\title{
Five Years Survival on Hemodialysis Predicted by Artificial Neural Network Model
}

\author{
Balsam Ahmed ${ }^{\mathrm{a} *}$, Borislav Dimitrov ${ }^{\mathrm{b}}$, Annalisa Perna ${ }^{\mathrm{b}}$, Giuseppe Remuzzi ${ }^{\mathrm{b}}$, Meguid El Nahas ${ }^{\mathrm{a}}$ \\ a. Sheffield Kidney Institute (SKI), Sheffield, UK \\ b. Mario Negri Institute for Pharmacological Research, Bergamo, Italy
}

\begin{abstract}
Introduction: Maintenance hemodialysis (HD) patients' morbidity and mortality remain unacceptably high. It is important to identify risk factors affecting outcome and define their relative contribution.

Methods: The data of 93 patients who started HD at the Sheffield Kidney Institute between January 1997 and June 1998 were analyzed retrospectively. Potential risk predictors of 5-year survival were evaluated both at baseline and at 1-year follow-up, including changes from baseline.
\end{abstract}

Results: Patients median age was 60 years (range: $27-80$ ), $75 \%$ were males and $23 \%$ were diabetics. According to the Index of Co-Existing Disease (ICED) score, 18\% of patients were level 0-1 (low comorbidity), 56\% level 2 and $26 \%$ level 3. The 5-year survival was $58 \%$. The Cox models identified eight independent time-adjusted risk factors for 5-year survival, the importance of which as independent predictors was confirmed by logistic regression models. According to the artificial neural network (ANN) models, the relative importance of these factors was as follows: age $(26.6 \%)$, baseline systolic blood pressure $(24.1 \%)$, mean Kt/V during the first year (19.7\%), baseline ICED score (14.8\%), baseline diastolic blood pressure $(8.6 \%)$, serum calcium change from baseline $(2.8 \%)$, blood urea change from baseline $(2.1 \%)$, serum creatinine change from baseline (1.3\%). This ANN model had a high level of predictive performance as assessed by accuracy (93.3\%) and by Receiver Operating Characteristic (ROC) curve analysis (AUC $=0.92$ ).

Conclusion: Integrated use of regression analysis and probabilistic models allow computation of individual risk of mortality in HD. This may help in optimizing care and costs.

\footnotetext{
* Corresponding Author; Assistant professor, Ahfad Medical School, Khartoum, Sudan

Email: balsamsator@hotmail.com
}

Key Words: hemodialysis, risk factors, ICED, artificial neural network

\section{Introduction}

The annual mortality rates in maintenance hemodialysis (HD) patients remain high, from $9 \%$ in Japan to $16 \%$ in Europe to $24 \%$ in the United States, on average. In the United States, only one third of dialyzed patients survive 5 years, and mortality of cardiovascular origin is 10-20 times greater than in the general population [1]. Comorbidity, advanced age and increased frequency of cardiovascular risk factors all contribute to outcome. It is now generally accepted that many factors leading to premature death in dialysis patients are already present when patients are started on renal replacement therapy (RRT). Among these, baseline congestive heart failure [2, 3], diabetes [4] and malnutrition [5] have strong impacts on prognosis. Even when these factors are taken into account, survival is still shorter in this particular group of individuals than in the normal population. Early identification of baseline risk factors that may be amenable to therapeutic intervention, has a greater practical attraction because their correction may reduce dialysis mortality.

A number of predictive analyses of outcome on HD have been undertaken over the years. Previous studies have, in general, assessed comorbidity at the start of dialysis treatment $[6,7]$ but there are very scarce data on the subsequent changes in comorbidity [8]. Whether changes in comorbidity are measurable by the current instruments and the extent to which changes in comorbidity alter the mortality-risk remain to be determined. The Artificial Neural Network (ANN) is a learning system based on a computational technique, which attempts to simulate the neurological processing ability of the brain [9]. Individual risk of progression to a given event can be effectively predicted by probabilistic tools such as artificial intelligence neural network models. These models offer an overall better performance than traditional statistical methods as they are not constrained by assumptions of specific relationships 
between dependent and independent variables. They can powerfully model the complex non-linear relationships in different subgroups of the population. However, the final output is strongly dependent on the choice of the input variables (risk factors). Potential input parameters can be assessed by expert opinions [9] or, better, through multifactorial analyses [10]. In addition to identifying factors significantly associated with a given outcome, the ANN can order risk factors according to their relative importance and is particularly effective when data are incomplete, imprecise or noisy [10].

Our aim in this study was to identify risk markers/factors for low 5-year survival on $\mathrm{HD}$, and to compare the performance of ANN and multivariate logistic regression models in the prediction of mortality in HD.

\section{Methods}

\section{Patients and Study design}

This is a single cohort, retrospective study that investigated the role of baseline and follow-up clinical and socio-demographic parameters in predicting the 5 -year survival of patients on HD. All patients who started HD at the Sheffield Kidney Institute (SKI) in the period between January 1997 and June 1998 and who have survived more than 90 days on HD were included in the analysis. Data were collected retrospectively from the hospital computer database and the patients' files, and included demographic (age, gender and race), baseline and follow-up clinical (causes of renal disease, comorbid conditions and causes of death) and biochemical (serum urea, creatinine, calcium, phosphorus, calciumphosphorus product, protein, albumin, hemoglobin, hematocrit and ferritin) data. Data were also collected on dialysis adequacy, including urea reduction ration (URR) and $\mathrm{Kt} / \mathrm{V}$, as well as on duration and causes of hospital admissions. Follow up data were collected at three months intervals for 5 years.

Potential predictors of 5-year survival were evaluated twice, at baseline and at 1-year follow-up. The relative change in biochemical parameters and comorbidity score during this period was also considered as a potential predictor of 5-year survival. The relative change was calculated according to the following equation:

Percent change $=[(1$-year value - baseline value $) /$ (baseline value)] X 100

\section{Comorbidity assessment (ICED score)}

Comorbidity is defined as a medical condition other than the primary disease. For the assessment of comorbidity in this study, we adapted the Index of Co-Existing Disease (ICED) score from the HEMO study [1] by excluding
HIV. The ICED scoring system is based on medical record review of 17 medical conditions summarized in the Index of Disease Severity (IDS) which ranges from $0-3$, and 11 physical functions summarized in the Index of Physical Impairment (IPI) which ranges from 0-2. The final ICED score which ranges from 0-3 is determined after assessment of the IDS and IPI scores.

\section{Statistical analysis}

The main outcome, 5-year patients' survival, and gender were considered as binary variables. The ICED score was employed as a numerical ordinal variable. All remaining variables were considered as continuous variables.

\section{Data management and the algorithm of the integrated approach}

Three groups of models were constructed to identify markers of survival in HD: (A) the first group of models assessed potential markers at baseline; (B) the second group of models assessed variables at 1-year follow up and included their relative change from baseline; and (C) the third group of models assessed a combination of the best independent predictors from group (A) and (B). Factors found at univariate analysis to be significantly associated with the outcome, which later retained an independent predictive value at the multivariate backward likelihood-ratio Cox regression analysis, were used to construct predictive optimal logistic regression (multivariate backwards likelihood-ratio) and/or ANN models.

\section{Artificial intelligence neural network modeling (ANN)}

Data were approximately partitioned in random into 3 sub-sets: $70 \%$ training set, $15 \%$ validation set and $15 \%$ test set. A class of ANN models was created and the best ANN model was chosen for training by exhaustive search algorithm (logistic activation function, cross-entropy error function and 0.5 accept level). The best network was created and trained with a built-in algorithm by the batch-back-propagation supervised method whereas the initial learning was set at 1.0 and the momentum at 0.8 , with dynamic adjustment during the iteration procedures. The relative importance of input factors in respect to the outcome (5-year survival on HD) was calculated using the sum of the absolute connection weights from each input node to the nodes of the next layer. The stopping role of learning during the training procedure was assumed as a state of maximum overall correctness of prediction (i.e. maximum correct classification rate [CCR]) of the validation set (pre-defined at a maximum of $95 \%$ ). The performance of ANN model was assessed by receiver operating characteristic (ROC) curve analysis 
Ahmed et al

Table 1: Baseline and 1-year follow up values of serum biochemistry and hematology parameters

\begin{tabular}{lll}
\hline Variable & Baseline & After one year \\
\hline Urea $(\mathrm{mmol} / \mathrm{l})$ & $38.4 \pm 13.43$ & $24.3 \pm 6.6$ \\
Creatinine $(\mu \mathrm{mol} / \mathrm{l})$ & $929 \pm 256$ & $929 \pm 287$ \\
$\mathrm{HCO}_{3}(\mathrm{mmol} / \mathrm{l})$ & $21 \pm 4.6$ & $23.7 \pm 3.4$ \\
$\mathrm{Ca}(\mathrm{mmol} / \mathrm{l})$ & $2.27 \pm 0.22$ & $2.4 \pm 0.2$ \\
$\mathrm{PO}_{4}(\mathrm{mmol} / \mathrm{l})$ & $2.15 \pm 0.63$ & $1.9 \pm 0.6$ \\
$\mathrm{Ca} \mathrm{X} \mathrm{PO}$ & $4.8 \pm 1.34$ & $4.5 \pm 1.4$ \\
Total protein $(\mathrm{g} / \mathrm{l})$ & $64 \pm 8.2$ & $66.4 \pm 6.6$ \\
$\mathrm{Albumin}(\mathrm{g} / \mathrm{l})$ & $34(19-45)$ & $36.9 \pm 5.1$ \\
$\mathrm{Urate}(\mu \mathrm{mol} / \mathrm{l})$ & $444(288-880)$ & $384 \pm 83$ \\
$\mathrm{Hb}(\mathrm{g} / \mathrm{l})$ & $9 \pm 1.3$ & $10.2 \pm 1.8$ \\
$\mathrm{HCT}(\%)$ & $27 \pm 0.04$ & $31 \pm 0.1$ \\
$\mathrm{ALP}(\mathrm{IU} / \mathrm{l})$ & $87(31-430)$ & $81(36-520)$ \\
Ferritin $(\mu \mathrm{g} / \mathrm{l})$ & $370(46-7686)$ & $421(35-2133)$ \\
$\mathrm{Kt} / \mathrm{V}$ & - & $1.2 \pm 0.1$ \\
\hline
\end{tabular}

Data were presented as mean $\pm \mathrm{SD}$; median (range) and number $(\%)$

which test for sensitivity, specificity and accuracy, on the whole dataset (overall) as well as on each of the 3 sub-sets (training, validation and test sets). The larger the area under the curve (AUC), the better is the model performance (pre-defined as being acceptable if above the level of 0.60). Finally, the best predictive model was chosen according to the performance criteria e.g., overall AUC and model accuracy.

\section{Results}

\section{Patients characteristics}

The total number of patients included in this analysis was 93. Median age was 60 years (range: $27-80$ ), $75 \%$ were males, $23 \%$ were diabetics and $80 \%$ were hypertensive. The 5-year survival was $58 \%$. The baseline and 1-year follow-up serum biochemistry and hematology parameters are shown (Table 1). There was a striking burden of comorbidity; $93.5 \%$ of patients were found to have at least one of the 17 disease conditions, $56 \%$ scored ICED level two and one quarter of the patients had ICED score of three, indicating symptomatic disease, the need for medications, or hospitalizations. The most prevalent disease was hypertension, present in more than $80 \%$ of patients, with $50 \%$ scored as ICED level two, indicating the need for medications. Cardiovascular disease was common, with $30 \%$ of patients having at least one or more of the four cardiac categories. On the second evaluation of comorbidity after one year in HD, $53 \%$ had no change in comorbidity score (ICED level), 31\% had one level increase and $10 \%$ had two level increase. On the other hand only $6 \%$ had one level decrease, this improvement was mainly due to better control of the blood pressure.

\section{Survival}

Half of the mortalities were due to cardiovascular causes $(51 \%)$. The second common cause of death was sepsis $(26 \%)$, followed by malignancy $(13 \%)$. The 5 -year cumulative survival functions, stratified by age or comorbidity levels, are presented (Figures 1 and 2). Patients who started dialysis at the age of 65 years or more had the highest mortality with a 5 -year cumulative survival of $65 \%$. Also, patients with severe comorbidity (ICED level 3) had the highest morality compared to other ICED levels with a 5-year cumulative survival of around $50 \%$.

\section{Survival and baseline parameters (group A models)}

Among baseline variables, only four variables were identified by the Cox model as significant time-to-death adjusted independent predictors of 5-year survival in HD (Table 2). The independent predictive role of these four baseline factors was further studied by logistic regression model; this model showed $79.6 \%$ sensitivity, $69.2 \%$ specificity and $75.3 \%$ accuracy (Table 3 ).

To increase this productivity, these factors were analysed further by an ANN model. Many network architectures were analyzed and evaluated using the complete dataset 
Figure 1: Cumulative 5-year survival curves stratified by age groups $(P=0.001)$

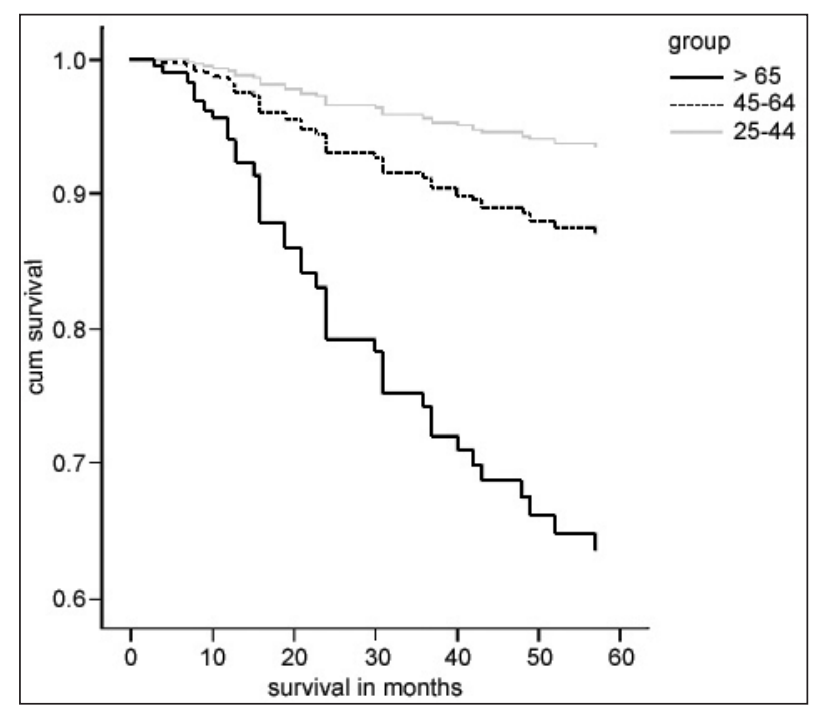

with these four identified predictors of survival used as input variables. The final architecture that reached optimal results within a state of higher accuracy and lowest errors to predict 5-year mortality consisted of three layers and 11 nodes, i.e. 4-6-1 design (Figure 3). This network showed the highest level of predictive performance as assessed by ROC curve analysis; the AUC was 0.90 with overall model accuracy of $82.2 \%$ (Table 4 ).

\section{Survival and 1-year follow-up parameters (group B models)}

The application of both Cox and logistic regression approaches neither produced significant models nor were they able to order the potential follow-up factors by their impact on survival. Therefore, all potential factors at 1-year follow-up, including relative percent change from baseline, were entered into an ANN model. The network was optimized out of 27 most simple possible architectures with three layers and produced the order of potential predictive importance of the studied factors. Only those with a relative importance above $1.5 \%$ were chosen for further analysis, i.e. serum creatinine change $(41.7 \%)$, hemoglobin change $(15.5 \%)$, serum phosphorus change $(13.6 \%)$, blood urea change $(12 \%)$, serum calcium change $(10.5 \%)$, ICED change $(2.8 \%)$ and serum albumin change $(1.5 \%)$.

\section{Survival and combination (baseline and 1 year follow up) predictors (group C models)}

We combined the 4 significant baseline predictors (group A model) with the follow up predictors (group B ANN model) and, together with $\mathrm{Kt} / \mathrm{V}$, we constructed a multivariate Cox model. The Cox model identified only
Figure 2: Cumulative 5-year survival curves stratified by ICED comorbidity-score levels $(P=0.000)$

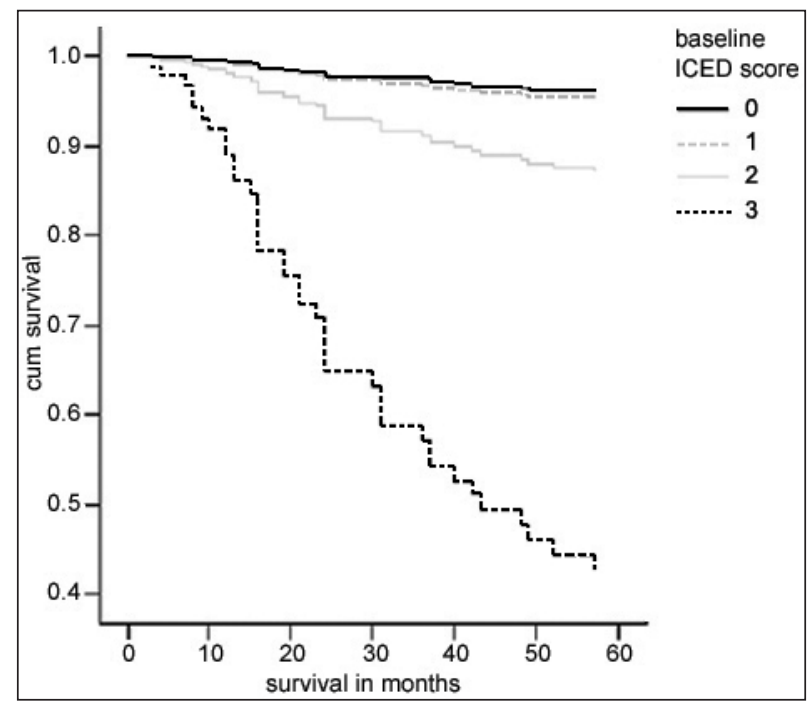

eight independent time-adjusted risk factors for 5-year survival (Table 5). These were entered into a logistic regression model which confirmed their importance as independent predictors of mortality in HD (Table 6), this model showed $83 \%$ sensitivity, $67.6 \%$ specificity and $76.7 \%$ accuracy. These factors were then entered into an ANN model and a number of network architectures were analyzed and evaluated using the complete dataset with the eight independent time-to-death adjusted factors. The final architecture that reached optimal results within a state of higher accuracy and lowest errors to predict 5-year mortality consisted of three layers and 15 nodes, 8-6-1 design, i.e. eight variables in the input layer, six nodes in the hidden layer and one node in the output layer (Figure 4). The performance of this network was assessed by ROC curve analysis which showed high level of predictive performance; the AUC was 0.94 and the overall accuracy was $93.3 \%$ (Table 7).

\section{Discussion}

This study investigated the impact of demographic, baseline and one year follow up clinical and biochemical parameters on five years mortality in HD. It showed that at the beginning of HD old age, level of comorbidity (ICED score), high diastolic blood pressure (DBP) and low systolic blood pressure (SBP) are significant predictors of 5-year survival on HD. In addition to these four baseline risk factors, this study showed that after the first year on $\mathrm{HD}$, low $\mathrm{Kt} / \mathrm{V}$, low relative change of serum creatinine from baseline, and high relative change in blood urea and calcium are important indicators for five years mortality. 
Table 2: Cox survival model with baseline predictors (group A)

\begin{tabular}{llllll}
\hline Variable & Unit of increase & P-value & (Exp.B) & \multicolumn{2}{c}{$\mathbf{9 5 \% \text { confidence level for OR }}$} \\
\cline { 5 - 6 } & & & Lower & Upper \\
\hline Old Age & 10 years & 0.000 & 1.07 & 1.037 & 1.103 \\
High ICED level & 1 level & 0.000 & 5.93 & 2.681 & 13.129 \\
SBP hypotension & $10 \mathrm{~mm} \mathrm{Hg}$ & 0.000 & 0.95 & 0.918 & 0.975 \\
DBP hypertension & $10 \mathrm{~mm} \mathrm{Hg}$ & 0.001 & 1.1 & 1.041 & 1.170 \\
\hline
\end{tabular}

Table 3: Logistic regression model with baseline predictors (group A)

\begin{tabular}{llllll}
\hline Variable & Unit of increase & P-value & (Exp.B) & \multicolumn{2}{c}{$\mathbf{9 5 \%}$ confidence level for OR } \\
\cline { 4 - 5 } & & & Lower & Upper \\
\hline Age & 10 years & 0.000 & 1.09 & 1.037 & 1.134 \\
ICED score & 1 level & 0.001 & 5.4 & 2.038 & 14.332 \\
SBP & $10 \mathrm{~mm} \mathrm{Hg}$ & 0.009 & 0.94 & 0.895 & 0.985 \\
DBP & $10 \mathrm{~mm} \mathrm{Hg}$ & 0.01 & 1.12 & 1.025 & 1.230 \\
\hline
\end{tabular}

We also found that an integrated approach based on a sequential use of Cox regression, logistic regression and probabilistic models (ANN) was effective in identifying and ordering risk factors for their relative importance in predicting mortality in HD. In this sense, a regression dilution effect in our multivariate models was unlikely to occur due to measurement variability. Moreover, the predictive importance of the factors from the multivariate regression was confirmed by the ANN modeling in which such a dilution effect could not be present.

Several countries have reported an increase in the age of patients starting RRT. According to the 2004 UK renal registry, the median age of patients started on RRT in UK was 64.8 years. In European limb of the DOPPS study the mean age was 60.2 years [12]. Old age is the most important of the demographic factors associated with increased morbidity and mortality in ESRD, and there are many reports showing that survival declines with increasing age [12-15]. This study is in agreement with previous reports that showed that old age is the most important independent risk factor for five years mortality in HD.

The presence of comorbid conditions is an increasingly common problem, being much more prevalent in new patients started on dialysis today than previously [6, 16-18]. Obviously, the concurrent presence of other lifethreatening conditions, as well as increased severity of comorbid conditions will also affect overall survival $[8$,
$19,20]$. Some studies have shown that the ICED score is a strong, independent predictor of death in dialysis patients. The risk of death was graded according to the incremental levels of the ICED score, and the results were consistent and independent of the size or setting of these studies. The relationship between the baseline ICED level and death remained significant even after adjustment for age, serum albumin, and diabetic status $[8,11]$. In this study, we also found that baseline comorbidity was a strong independent predictor of mortality even after adjustment for other risk factors. Moreover, we found that graded levels of the ICED score were incrementally associated with mortality risk.

This analysis also evaluated the change in comorbidity over time, utilizing the ICED score. However, changes in the ICED level from baseline did not add to the baseline level in prediction of subsequent mortality. This contradicts the report of Miskulin and his group who found the changes in comorbidity to be a sensitive predictor of mortality [8]. However, they measured changes of comorbidity over two years, while we had a follow up period of only one year. The power of our study may have been inadequate to reproduce such an association using multivariate analysis, and perhaps longer follow-up is needed to demonstrate the effect of comorbidity changes on mortality.

Dialysis adequacy is not easy to quantify, and commonly used parameters include control of fluid overload and 
Figure 3: ANN model with baseline predictors (group A model)

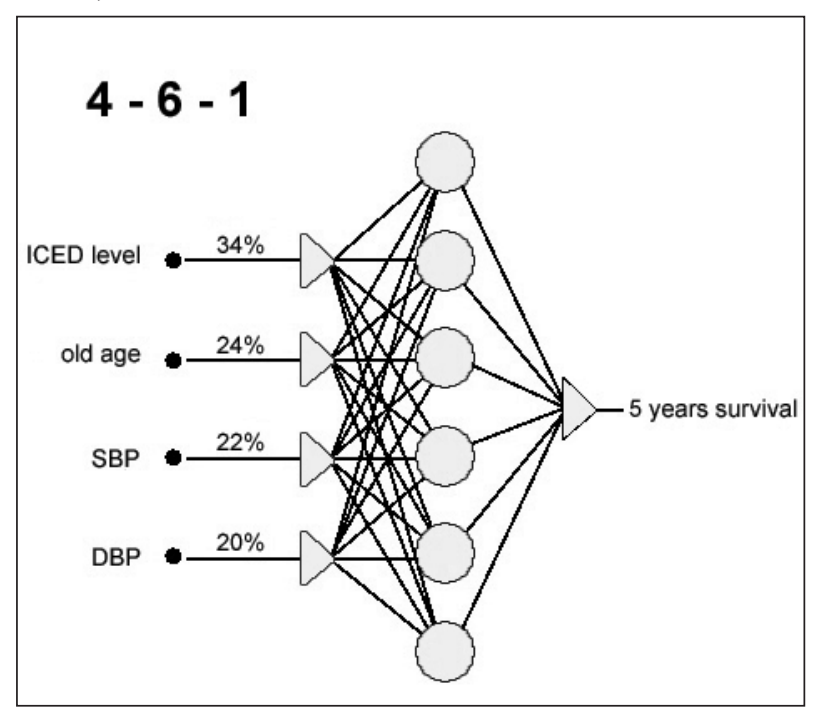

The final architecture that reached optimal results consisted of 3 layers and 11 nodes (4-6-1 design), i.e, 4 variables (grey triangles on the left) in the input layer, 6 nodes (grey circles) in the hidden layer and one node (grey triangle on the right) in the output layer

electrolyte disturbances, correction of metabolic acidosis and the dialysis dose. The most frequently used measure of the delivered dialysis dose is $\mathrm{Kt} / \mathrm{V}$. To date, there is a debate concerning the target dialysis dose that should be reached to ensure the best patient outcome [21, 22]. The most recent K-DOQI guidelines [23] recommend a minimum single-pool Kt/V of 1.2, roughly corresponding to a URR of $65 \%$, for thrice weekly HD in patients with a residual glomerular filtration rate of less than $2 \mathrm{ml} / \mathrm{min}$ per $1.73 \mathrm{~m}^{2}$. The European Best Practice Guidelines of 2002 recommend higher values: double-pool Kt/V of at least 1.2 and single-pool Kt/V of at least 1.4 [24].

A number of observational studies have shown that single-pool $\mathrm{Kt} / \mathrm{V}$ is independently associated with patient survival $[7,25]$. This report, in agreement with previous reports, showed a $9.9 \%$ decreased risk of mortality for each 0.1 increase in the mean $\mathrm{Kt} / \mathrm{V}$ during the first year of HD. It's worth mentioning that we did not evaluate the effect of higher doses of dialysis on patients' survival as we were limited by the number of patients included in this analysis.

The relationship between $\mathrm{BP}$ and the mortality in dialysis patients remains controversial. There has been considerable discussion regarding whether hypertension is a risk factor for mortality or, in reverse, is associated with a decreased risk of mortality, as well as to the extent to which BP must be lowered to minimize mortality. Some authors argue that low normal BP values are associated with increased mortality and that moderate hypertension provides optimal survival [26-28]. Whether low BP values simply indicate high comorbidity scores or directly reduce life expectancy remains unclear. In the dialysis population, a significant correlation between low predialysis SBP and mortality has been reported by several studies based on large cohorts of patients [12, 28, 29], and our finding agree with these reports. This probably reflects advanced cardiovascular disease (CVD), since low BP values often characterize patients with poor cardiac function and high risk of cardiovascular death. Another potential confounding factor could be that patients with low pre-dialysis systolic BP may receive shorter dialysis sessions, or that they are at increased risk of intradialytic hypotension. Indeed, the risk of death among high risk diabetic hemodialysis patients was increased by a factor of 3 when two or three hypotensive episodes occurred per week [29]. Even if frank hypotensive episodes do not occur, it is plausible that massive sympathetic activation is provoked by ultrafiltration in patients with pre-existing cardiac disease, and this is a deleterious factor contributing to cardiac arrhythmia and cardiac ischemia. However, we did not observe the U-shaped curve relationship between SBP and mortality that has been mentioned in previous studies $[27,28]$.

In the present investigation high DBP was found to be an independent risk factor for mortality. The risk of death was increased by $9 \%$ per one $\mathrm{mmHg}$ increase in the DBP. However, some authors found that low rather than high DBP predicts mortality [30]. A third group of authors described a U- shape relationship between DBP

Table 2: Cox survival model with baseline predictors (group A)

\begin{tabular}{llllll}
\hline Sets & Sensitivity (\%) & Specificity (\%) & Accuracy (\%) & PPV (\%) & NPV (\%) \\
\hline Training & 80 & 81.1 & 80.6 & 74 & 87 \\
Validation & 100 & 90 & 92.9 & 80 & 100 \\
Test & 67 & 86 & 78.6 & 80 & 78 \\
Overall & 80 & 84 & 82.2 & 76 & 87 \\
\hline
\end{tabular}

PPV: positive predictive value, NPV: negative predictive value 
Table 5: Cox survival model with baseline and 1-year follow-up predictors (group C)

\begin{tabular}{llllll}
\hline Variable & Unit of increase & P-value & (Exp.B) & \multicolumn{2}{c}{$\mathbf{9 5 \% \text { confidence level for OR }}$} \\
\cline { 5 - 5 } & & & Lower & Upper \\
\hline Age & 10 years & 0.000 & 1.07 & 1.034 & 1.102 \\
ICED score & 1 level & 0.000 & 5.69 & 2.515 & 12.856 \\
Kt/V in the first year & 0.1 & 0.000 & 0.007 & 0.001 & 0.097 \\
DBP & $10 \mathrm{mmHg}$ & 0.006 & 1.094 & 1.026 & 1.165 \\
SBP & $10 \mathrm{mmHg}$ & 0.02 & 0.96 & 0.932 & 0.993 \\
Blood urea change & $1 \%$ & 0.01 & 1.02 & 1.002 & 0.994 \\
Serum creatinine change & $1 \%$ & 0.002 & 0.98 & 0.962 & 2.175 \\
Serum calcium change & $1 \%$ & 0.059 & 1.46 & 0.985 & \\
\hline
\end{tabular}

Table 6: Logistic regression model with baseline and 1-year follow-up predictors (group C)

\begin{tabular}{|c|c|c|c|c|c|}
\hline \multirow[t]{2}{*}{ Variable } & \multirow[t]{2}{*}{ Unit of increase } & \multirow[t]{2}{*}{ P-value } & \multirow[t]{2}{*}{ (Exp.B) } & \multicolumn{2}{|c|}{$95 \%$ confidence level for OR } \\
\hline & & & & Lower & Upper \\
\hline Age & 10 years & 0.000 & 1.12 & 1.053 & 1.185 \\
\hline ICED score & 1 level & 0.000 & 8.74 & 2.717 & 28.115 \\
\hline $\mathrm{Kt} / \mathrm{V}$ in the first year & 0.1 & 0.001 & 0.001 & 000 & 0.041 \\
\hline Serum creatinine change & $1 \%$ & 0.008 & 0.97 & 0.949 & 1.000 \\
\hline DBP & $10 \mathrm{mmHg}$ & 0.04 & 1.13 & 1.007 & 1.258 \\
\hline SBP & $10 \mathrm{mmHg}$ & 0.04 & 0.94 & 0.888 & 0.998 \\
\hline Blood urea change & $1 \%$ & 0.05 & 1.04 & 1.010 & 1.068 \\
\hline Serum calcium change & $1 \%$ & 0.054 & 1.83 & 0.990 & 3.399 \\
\hline
\end{tabular}

and survival in HD [28]. Indeed, high DBP is a risk factor for CVD and late mortality, while low DBP identifies patients with advanced CVD and hence implicates a higher risk of early mortality.

Although this study failed to demonstrate some of the previously reported associations between baseline biochemical parameters and survival, it has clearly shown that changes of some of these parameters (serum creatinine, blood urea and serum calcium) during the follow-up period had a negative impact on survival. In dialysis patients, the serum creatinine concentration relates to nutritional status and reflects somatic protein stores, muscle mass and dietary protein intake. Hence, low serum creatinine levels have been shown to be highly predictive of mortality in HD patients [31-33]. As confirmed before, mortality risk in this study was associated with lower relative change in serum creatinine levels after one year, that is, less increase in serum creatinine level over one year of follow-up. Each 1\% increase in the relative change of serum creatinine level was associated with $3 \%$ increase in survival. Low serum creatinine levels reflect small muscle mass or malnutrition; factors which have been shown to be associated with comorbidity [7, 11, 31-35]. It is worth noting that the change in serum creatinine level over time may be more important than absolute values, since well-nourished well-dialyzed patients may have relatively lower serum creatinine levels than similar patients undergoing inadequate dialysis.

Urea is the most frequently used marker in measures of dialysis adequacy. The national co-operative dialysis study (NCDS) reported that blood urea nitrogen (BUN) was an important determinant of morbidity in patients undergoing HD [21]. Later, the same group found a Ushaped association between BUN and survival in HD [31]. They speculate that the higher mortality in the high BUN group is due to relative under-dialysis, while the higher mortality in the low BUN group may be due to under-nutrition. In this cohort, higher relative change of blood urea level after one year, that is a greater increase in blood urea levels after the first year, is a significant risk of mortality, with a $4 \%$ increase in the risk of death for each $1 \%$ increase in the relative change of the blood 
Figure 4. ANN model with combined baseline and 1-year follow-up predictors (group C models)

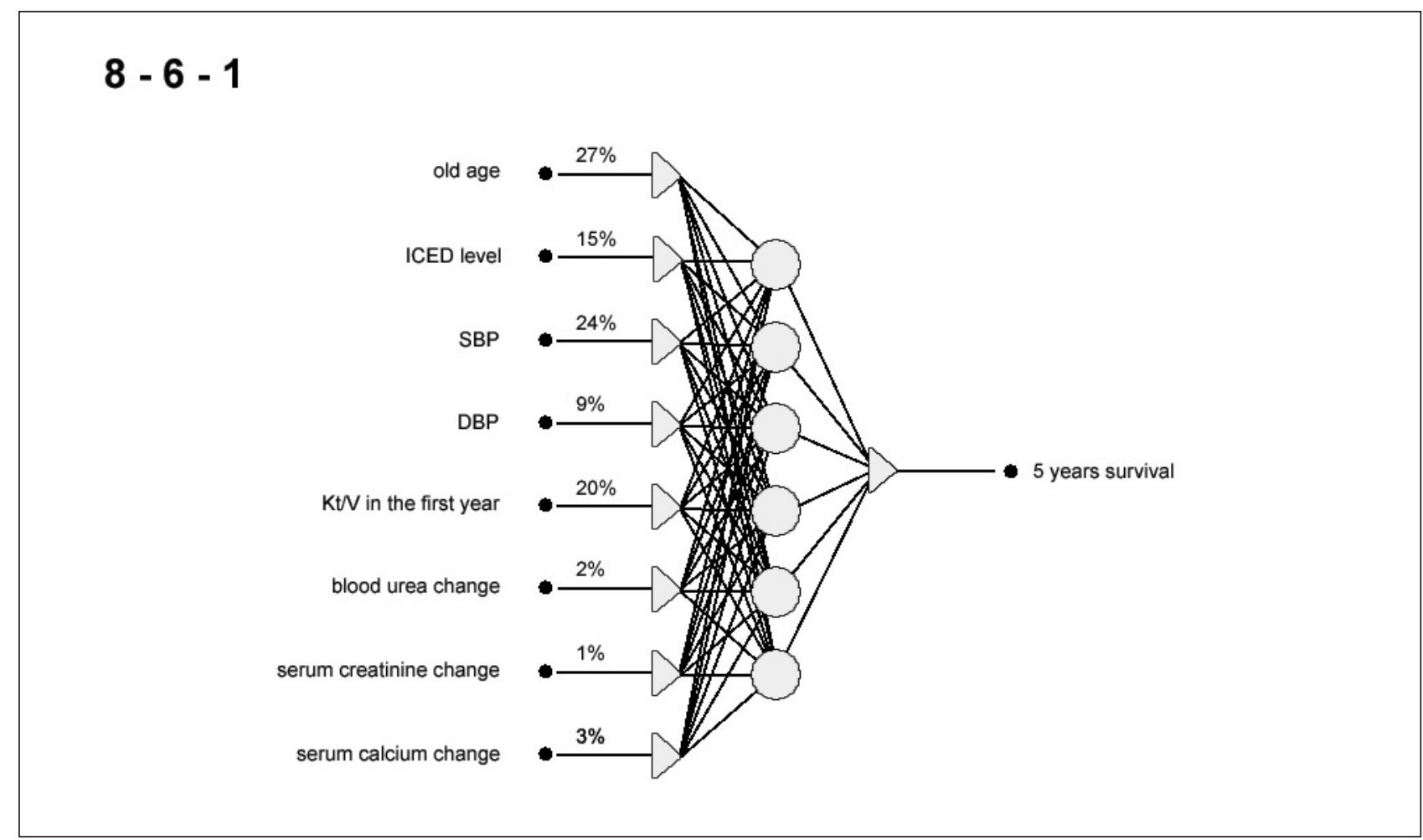

The final architecture that reached optimal results consisted of 3 layers and 15 nodes (8-6-1 design), i.e. 8 variables (grey triangles on the left) in the input layer, 6 nodes (grey circles) in the hidden layer and one node (grey triangle on the right) in the output layer

urea. It is possible that high blood urea level acted as an indicator of inadequate dialysis.

Previous reports have identified associations between certain disorders of mineral metabolism and all-cause and CVD mortality in HD. In the present study, high relative change in serum calcium level from baseline, i.e. a greater increase in serum calcium level after one year on dialysis, was a significant independent predictor of mortality (RR of 1.46). These results contrast with results reported earlier by Foley and colleagues, who found that low serum calcium $(<8.8 \mathrm{mg} / \mathrm{dl})$ was associated with increased mortality [35].

We found no association between mortality and some of the well established risk factors, i.e. serum albumin and phosphate levels, calcium phosphate product, hemoglobin, hematocrit, body mass index and smoking. It is possible that these factors simply identify less healthy patients and serve as a convenient clinical surrogate for comorbidity assessment, rather than having a direct causal relationship to survival.

A major strength of the present study is the integrated approach, with sequential use of univariate and multivariate Cox regression analysis and the ANN models. This might lead to the development of optimum prediction models. A regression dilution effect in this multivariate model was unlikely to occur due to measurement variability. Moreover, the predictive importance of the explanatory factors from the multivariate regression was confirmed by the ANN modeling in which such a dilution effect could not be present. This approach is flexible and it can be adopted by physicians and policy makers in order to optimize the performance of the model.

This study also compared the performance of ANN models against logistic regression models in predicting mortality in HD. Both logistic regression and the ANN models did well in predicting mortality in HD. However, ROC curve analysis proved that ANN models significantly outperformed logistic models in all aspects; sensitivity, specificity and accuracy. Currently, the logistic regression and the ANN are the most widely used models in outcome studies. One of the advantages of ANN analysis is that it allows the inclusion of a large number of variables and can deal with non-normally distributed data [36]. Another advantage of the ANN approach is that the ANN is not constrained by predefined assumptions between the dependent and independent variables and can powerfully model complex non-linear relationships [36]. One of the strengths of the ANN is its ability to find patterns despite missing data [36]. Neural networks have perhaps a 
Ahmed et al

Table 7: Predictive performance of combined baseline and 1-year follow-up predictors (group C) ANN model

\begin{tabular}{llllll}
\hline Sets & Sensitivity (\%) & Specificity (\%) & Accuracy (\%) & PPV (\%) & NPV (\%) \\
\hline Training & 90.3 & 96.7 & 93.5 & 96.6 & 91 \\
Validation & 80 & 88.9 & 85.7 & 80 & 88.9 \\
Test & 60 & 100 & 85.7 & 100 & 82 \\
Overall & 85.4 & 96 & 93.3 & 95 & 87 \\
\hline
\end{tabular}

PPV: positive predictive value, NPV: negative predictive value

special appeal to the medical community because of their superficial resemblance to the human brain, a structure with which most physicians are comfortable, and seem to promise prediction without the difficulties associated with use of mathematics [36].

\section{Conclusion}

A high ICED score, old age, low SBP and high DBP at commencing $\mathrm{HD}$ predicted low 5-year survival rates. In addition, the percent changes from baseline in serum calcium, blood urea, serum creatinine as well as inadequate dialysis during the first year on HD were independent predictors of 5-year mortality. The individual mortality risk index, computed by the integrated use of regression analysis and probabilistic (ANN) models, allowed prediction of outcome using a combination of factors and could provide useful information on healthassociated issues such as mortality, quality of life or cost of treatment. Such models also help to identify high risk subjects in order to intensify monitoring, prevention and treatment protocols, with the final goal of optimizing care and minimizing costs.

\section{References}

1. Foley RN, Parfrey PS, Sarnak MJ. Clinical epidemiology of cardiovascular disease in chronic renal disease. Am J Kidney Dis. 1998 Nov;32(5 suppl 3):S112-19.

2. Foley RN, Parfrey PS, Harnett JD, Kent GM, Martin CJ, Murray DC, Barre PE. Clinical and echocardiographic disease in patients starting end-stage renal disease therapy. Kidney Int. 1995 Jan;47(1):186-92.

3. Harnett JD, Foley RN, Kent GM, Barre PE, Murray D, Parfrey PS. Congestive heart failure in dialysis patients: prevalence, incidence, prognosis and risk factors. Kidney Int. 1995 Mar;47(3):884-90.

4. Chantrel F, Enache I, Bouiller M, Kolb I, Kunz K, Petitjean P, Moulin B, Hannedouche T. Abysmal prognosis of patients with type 2 diabetes entering dialysis. Nephrol Dial Transplant. 1999 Jan;14(1):129-36.
5. De Lima JJ, da Fonseca JA, Godoy AD. Baseline variables associated with early death and extended survival on dialysis. Ren Fail. 1998 Jul;20(4):581-7.

6. Mailloux LU, Napolitano B, Bellucci AG, Mossey RT, Vernace MA, Wilkes BM. The impact of co-morbid risk factors at the start of dialysis upon the survival of ESRD patients. ASAIO J. 1996 May-Jun;42(3):164-9.

7. Collins AJ, Ma JZ, Umen A, Keshaviah P. Urea index and other predictors of hemodialysis patient survival. Am J Kidney Dis. 1994 Feb;23(2):272-82.

8. Miskulin DC, Meyer KB, Martin AA, Fink NE, Coresh J, Powe NR, Klag MJ, Levey AS; Choices for Healthy Outcomes in Caring for End-Stage Renal Disease (CHOICE) Study. Comorbidity and its change predict survival in incident dialysis patients. Am J Kidney Dis. 2003 Jan;41(1):149-61.

9. Bohanec M, Zupan B, Rajkovic V. Applications of qualitative multi-attribute decision models in health care. Int J Med Inform. 2000 Sep;58-59:191-205.

10. Resnic FS, Popma JJ, Ohno-Machado L. Development and evaluation of models to predict death and myocardial infarction following coronary angioplasty and stenting. Proc AMIA Symp. 2000;690-3.

11. Miskulin DC, Athienites NV, Yan G, Martin AA, Ornt DB, Kusek JW, Meyer KB, Levey AS; Hemodialysis (HEMO) Study Group. Comorbidity assessment using the Index of Coexistent Diseases in a multicenter clinical trial. Kidney Int. 2001 Oct;60(4):1498-510.

12. Goodkin DA, Bragg-Gresham JL, Koenig KG, Wolfe RA, Akiba T, Andreucci VE, Saito A, Rayner HC, Kurokawa K, Port FK, Held PJ, Young EW. Association of comorbid conditions and mortality in hemodialysis patients in Europe, Japan, and the United States: the Dialysis Outcomes and Practice Patterns Study (DOPPS). J Am Soc Nephrol. 2003 Dec;14(12):3270-7.

13. Charra B, Calemard E, Ruffet M, Chazot C, Terrat JC, Vanel T, Laurent G. Survival as an index of adequacy of dialysis. Kidney Int. 1992 May;41(5):1286-91. 
14. Byrne C, Vernon P, Cohen JJ. Effect of age and diagnosis on survival of older patients beginning chronic dialysis. JAMA. 1994 Jan 5;271(1):34-6.

15. Mailloux LU, Bellucci AG, Napolitano B, Mossey T, Wilkes BM, Bluestone PA. Survival estimates for 683 patients starting dialysis from 1970 through 1989: identification of risk factors for survival. Clin Nephrol. 1994 Aug;42(2):127-135.

16. Collins AJ, Hanson G, Umen A, Kjellstrand C, Keshaviah P. Changing risk factor demographics in end-stage renal disease patients entering hemodialysis and the impact on long-term mortality. Am J Kidney Dis. 1990 May;15(5):422-32.

17. Merkus MP, Jager KJ, Dekker FW, de Haan RJ, Boeschoten EW, Krediet RT. Predictors of poor outcome in chronic dialysis patients: The Netherlands Cooperative Study on the Adequacy of Dialysis. The NECOSAD Study Group. Am J Kidney Dis. 2000 Jan;35(1):69-79.

18. Wallen MD, Radhakrishnan J, Appel G, Hodgson ME, Pablos-Mendez A. An analysis of cardiac mortality in patients with new-onset end-stage renal disease in New York State. Clin Nephrol. 2001 Feb;55(2):101-8.

19. Beddhu S, Bruns FJ, Saul M, Seddon P, Zeidel ML. A simple comorbidity scale predicts clinical outcomes and costs in dialysis patients. Am J Med. 2000 Jun 1;108(8):609-13.

20. Davies SJ, Phillips L, Naish PF, Russell GI. Quantifying comorbidity in peritoneal dialysis patients and its relationship to other predictors of survival. Nephrol Dial Transplant. 2002 Jun;17(6):1085-92.

21. Lowrie EG, Teehan BP. Principles of prescribing dialysis therapy: implementing recommendations from the National Cooperative Dialysis Study. Kidney Int Suppl. 1983 Apr;(13)S113-22.

22. Eknoyan G, Beck GJ, Cheung AK, Daugirdas JT, Greene T, Kusek JW, Allon M, Bailey J, Delmez JA, Depner TA, Dwyer JT, Levey AS, Levin NW, Milford E, Ornt DB, Rocco MV, Schulman G, Schwab SJ, Teehan BP, Toto R; Hemodialysis (HEMO) Study Group. Effect of dialysis dose and membrane flux in maintenance hemodialysis. N Engl J Med. 2002 Dec 19;347(25):2010-9.

23. Hemodialysis Adequacy 2006 Work Group. Clinical practice guidelines for hemodialysis adequacy, update 2006. Am J Kidney Dis. 2006 Jul;48 Suppl 1:S2-90.

24. Tattersall J, Martin-Malo A, Pedrini L, Basci A, Canaud B, Fouque D, Haage P, Konner K, Kooman J, Pizzarelli F, Tordoir J, Vennegoor M, Wanner C, ter Wee $\mathrm{P}$, Vanholder R. EBPG guideline on dialysis strategies. Nephrol Dial Transplant. 2007 May;22 Suppl 2:ii5-21.
25. Held PJ, Port FK, Wolfe RA, Stannard DC, Carroll CE, Daugirdas JT, Bloembergen WE, Greer JW, Hakim RM. The dose of hemodialysis and patient mortality. Kidney Int. 1996 Aug;50(2):550-6.

26. Salem MM. Hypertension in the hemodialysis population: a survey of 649 patients. Am J Kidney Dis. 1995 Sep;26(3):461-8.

27. PortFK, Hulbert-Shearon TE, Wolfe RA, Bloembergen WE, Golper TA, Agodoa LY, Young EW. Predialysis blood pressure and mortality risk in a national sample of maintenance hemodialysis patients. Am J Kidney Dis. 1999 Mar;33(3):507-17.

28. Zager PG, Nikolic J, Brown RH, Campbell MA, Hunt WC, Peterson D, Van Stone J, Levey A, Meyer KB, Klag MJ, Johnson HK, Clark E, Sadler JH, Teredesai P. "U" curve association of blood pressure and mortality in hemodialysis patients. Medical Directors of Dialysis Clinic, Inc. Kidney Int. 1998 Aug;54(2):561-9.

29. Koch M, Thomas B, Tschope W, Ritz E. Survival and predictors of death in dialysed diabetic patients. Diabetologia. 1993 Oct;36(10):1113-7.

30. Iseki K, Miyasato F, Tokuyama K, Nishime K, Uehara H, Shiohira Y, Sunagawa H, Yoshihara K, Yoshi S, Toma S, Kowatari T, Wake T, Oura T, Fukiyama K. Low diastolic blood pressure, hypoalbuminemia, and risk of death in a cohort of chronic hemodialysis patients. Kidney Int. 1997 Apr;51(4):1212-7.

31. LowrieEG, Lew NL. Death risk in hemodialysis patients: the predictive value of commonly measured variables and an evaluation of death rate differences between facilities. Am J Kidney Dis. 1990 May;15(5):458-82.

32. Avram MM, Bonomini LV, Sreedhara R, Mittman N. Predictive value of nutritional markers (albumin, creatinine, cholesterol, and hematocrit) for patients on dialysis for up to 30 years. Am J Kidney Dis. 1996 Dec;28(6):910-7.

33. Pifer TB, McCullough KP, Port FK, Goodkin DA, Maroni BJ, Held PJ, Young EW. Mortality risk in hemodialysis patients and changes in nutritional indicators: DOPPS. Kidney Int. 2002 Dec;62(6):2238-45.

34. Owen WF Jr., Lew NL, Liu Y, Lowrie EG, Lazarus JM. The urea reduction ratio and serum albumin concentration as predictors of mortality in patients undergoing hemodialysis. N Engl J Med. 1993 Sep 30;329(14):1001-6.

35. Foley RN, Parfrey PS, Harnett JD, Kent GM, Hu L, O'Dea R, Murray DC, Barre PE. Hypocalcemia, morbidity, and mortality in end-stage renal disease. Am J Nephrol. 1996;16(5):386-93.

36. Tu JV. Advantages and disadvantages of using artificial neural networks versus logistic regression for predicting medical outcomes. J Clin Epidemiol. 1996 Nov;49(11):1225-31. 